\title{
THE EXPERIMENTAL VERIFICATION OF A GENERALIZED MODEL OF EQUIVALENT CIRCUITS
}

\author{
ZOLTÁN LUKÁCS*1, DÁVID BACCILIERI ${ }^{1}$, AND TAMÁS KRISTÓF ${ }^{1}$ \\ ${ }^{1}$ Center for Natural Sciences, University of Pannonia, Egyetem u. 10, 8200 Veszprém, HUNGARY
}

\begin{abstract}
The determination of typical parameters of electrochemical systems, e.g. the polarization or charge transfer resistances, can be critical with regard to the application of Electrochemical Impedance Spectroscopy (EIS) if the lower frequency range is biased as a result of transport and/or adsorption/desorption processes. In such cases, the charge transfer resistance should be assessed from the higher frequency range which is typically inadequate in itself as an input for nonlinear parameter fitting. In earlier publications, an alternative mathematical treatment of both the Equivalent Circuit (EC) and of the parameter dispersion was provided using a generalized model of ECs and also a dispersion-invariant model of the electrochemical interface. In the present work, the previously presented experimental EIS results were crosschecked to verify the performance of the generalized model against a series of redox and corrosion systems. The results proved that the applied method is consistent and provides a fairly good correlation between the principal resistance data assessed by different methods.
\end{abstract}

Keywords: Electrochemical Impedance Spectroscopy, parameter dispersion, linearized model, equivalent circuit, polarization resistance

\section{Introduction}

Electrochemical Impedance Spectroscopy (EIS) is used to study systems in many electrochemical fields, e.g., electrode kinetics, the testing of bilayer systems, batteries, galvanic cells, corrosion, solid-state electrochemical processes, bioelectrochemistry, photovoltaic systems etc. In EIS, the studied system is perturbed from its equilibrium (or stationary) state by a small-amplitude sinusoidal potential signal. For this AC potential signal, it is assumed that the transfer function of the electrochemical kinetic system can be represented by a so-called equivalent circuit (EC). The simplest circuit of this kind is the Voigt circuit, a parallel RC circuit, complete with a resistor representing the pure ohmic ${ }^{\dagger}$ solution resistance, which approximates the transfer function of the electrochemical system well in many cases ${ }^{\ddagger}$ (see Fig. 1A).

The transfer function of Model A in Figure 1 is given by

$$
Z(\omega)=R_{\mathrm{S}}+\frac{R_{\mathrm{CT}}}{1+i \omega \tau},
$$

where $i$ denotes the imaginary unit, $\omega$ stands for the angular frequency and $\tau=R_{\mathrm{CT}} C_{\mathrm{DL}}$ represents the time constant of the RC circuit. Eq. 1 describes an ideal theoretical

\footnotetext{
*Correspondence: lukacs.zoltan@mk.uni-pannon.hu

$\dagger$ Pure ohmic in the electrochemically relevant frequency range.

${ }^{\ddagger}$ In this section, the effects of the transport and adsorption/desorption steps are not considered. They are mentioned in sufficient detail in the section where the experimental results are discussed.
}

model and the EIS curves plotted from it yield a perfect semicircle on the so-called Nyquist diagram. The value of the ohmic solution resistance $\left(R_{\mathrm{S}}\right)$ is determined from the high-frequency data $(\sim 10 \mathrm{kHz}-1 \mathrm{kHz})$. If diffusion, adsorption or other processes do not influence the curve, then the sum of the solution and charge transfer resistances can be determined from the low-frequency data. In contrast with the theoretical expectations, in practice, the impedance spectroscopy diagrams measured have always been slightly flattened ('depressed semicircles'). This depression can be modelled phenomenologically by using a power of less than 1 in the frequency-dependent term of Eq. 1:

$$
Z(\omega)=R_{\mathrm{S}}+\frac{R_{\mathrm{CT}}}{1+(i \omega \tau)^{1-\beta}},
$$

where $0<\beta \ll 1$ denotes the constant phase coefficient and the resulting element with the fractional power in Eq. 2 is referred to in the literature as a constant phase element. As the fractional power expression results in a constant phase impedance at high frequencies, this phenomenon is commonly referred to as the Constant Phase Element (CPE).

Research into the CPE and related phenomena dates back many decades. Cole and Cole $[1,2]$ investigated the capacitance of solid and liquid dielectrics as well as interpreted the appearance of the CPE as dependent on the frequency of the dielectric constant. The application of AC methods and EIS in particular to a wide range of electro- 


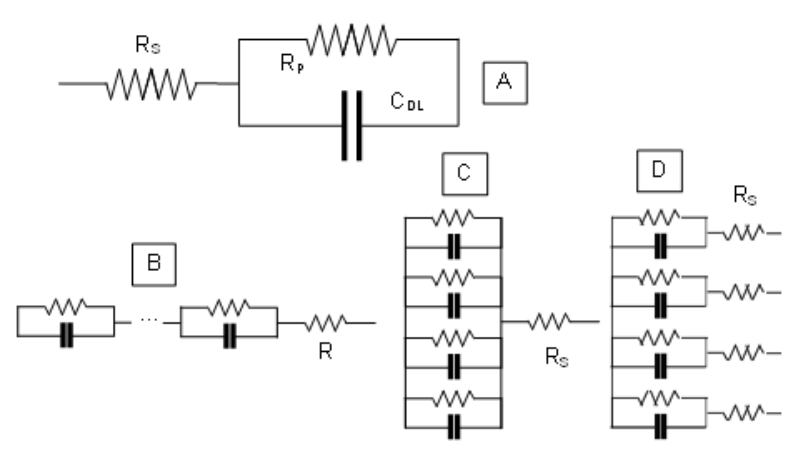

Figure 1: Equivalent circuits corresponding to Models A$\mathrm{D}$ and frequently applied in the relevant literature (see further comments in the text).

chemical systems has become quite common in electrochemical kinetics [3-8], electrode surface structure investigations [9-14], corrosion studies [15-21], battery and fuel cell development [22-26], membrane studies [27,28] and many other fields in electrochemistry.

The CPE appears in all the cited works and can be regarded with a high degree of certainty as an inherent characteristic of electrochemical systems. However, the origin and evaluation of the CPE has remained a controversial issue. Most works on the topics agree that the CPE can be interpreted as a consequence of the distribution of the time constant of RC circuits representing the characteristics with regard to the capacitance and conductivity of the electrochemical interface. Cole [1] assumes a lognormal distribution of the relaxation time constants. A similar distribution function is proposed by Brug [3]. In subsequent works, the appearance of the CPE is frequently interpreted by means of equivalent circuits that include many RC elements in parallel or series. (Implicitly, the distribution functions can also be recurred to such equivalent circuits.)

The applicable equivalent circuits can be roughly divided into two categories. Some fall into the category of the so-called '3D' (three-dimensional) dispersion models which correspond to the structures perpendicular to the surface, contributing to the conventional equivalent circuit with a series of additional parallel RC circuits $[14,15]$, as shown in Model B in Fig. 1. The 3D model (or its derivatives, see below) has been successfully applied in the description of passive films and coatings [29-31]. The so-called 2D dispersion models [3,14] are intended to describe the distribution of the EIS parameters in two dimensions on the electrode surface. This dispersion can be attributed to the heterogeneity of the surface and the resulting deviations of the (intensive) kinetic parameters, i.e., the time constants.

Discussions concerning the 2D dispersion can lead, however, to an unexpected conclusion if this is consequently carried out. The 2D dispersion model, i.e., the dispersion of the time constants of the RC circuits, representing the kinetic parameters of the individual active re- action sites connected in parallel to each other, as shown in Model C in Fig. 1, will degenerate to Model A. In order to avoid this, the effect of the solution resistance is taken into account to re-establish the ability of conventional EC patterns to interpret CPE behaviour ('While an Ohmic resistance in physical systems cannot be avoided, the example illustrated in Fig. 1B illustrates the crucial role played by the Ohmic resistance in CPE behavior associated with surface distributions.' [14]). However, there are at least three very important reasons to have serious reservations with regard to this concept. Firstly, Pajkossy has shown [10] that 'capacitance dispersion due to irregular geometry appears at much higher frequencies than is usual in electrochemical methodologies' which means that the irregular geometry, which acts on the EIS impedance function through the variation in the (local) solution resistance, cannot be the reason for the dispersion in the generally applied and electrochemically relevant frequency range. Secondly, in highly conductive solutions and/or with high-resistance (i.e., slow) electrochemical reactions, the contribution of the solution resistance to the overall impedance can be negligible compared to that of the charge transfer resistance, especially at lower frequencies. Thirdly, strictly speaking, the solution resistance is not an inherent part of the impedance of the electrochemical impedance system. By moving the tip of the Luggin capillary closer or farther away, the value of the measured solution resistance can be varied significantly, therefore, any calculations using it in the modelling or evaluation of the kinetic process are debatable.

In conclusion, Models $\mathrm{C}$ and $\mathrm{D}$ in Fig. 1 are not applicable in the interpretation of the CPE phenomenon. Consequently, by proceeding forwards on this path, it follows that Model A in Fig. 1 is not and cannot be the ultimate model (transfer function) of the electrochemical interface. The ultimate model is something more complex, which can, in certain cases, be simplified to Model A as far as the accuracy of measured data is concerned. In an earlier paper [32], this issue was already discussed in brief (see Fig. 3 and the relevant text in the cited paper).

This contradiction was realized in the relevant literature decades ago. In a noteworthy work, Agarwal et al. [33] approximated a number of ECs to Model B which obviously does not match the physical content of the approximated ECs, however, the fitted curves match the experimental ones very well. These results are also confirmed from another point of view. It has been proven $[34,35]$ that some ECs, exhibiting quite different elements and connection patterns, have the same transfer function. These findings also show that the concept of the 'equivalent circuit' is by no means as solid nor unambiguous as it would seem to be at first sight. After all, the question what is the minimum statement that can be both relevant and unambiguous concerning the EIS equivalent circuit and parameter determination in general is raised.

A possible answer to this question was presented in two recent publications $[36,37]$. It was assumed that Model A in Fig. 1 is approximately correct and devia- 

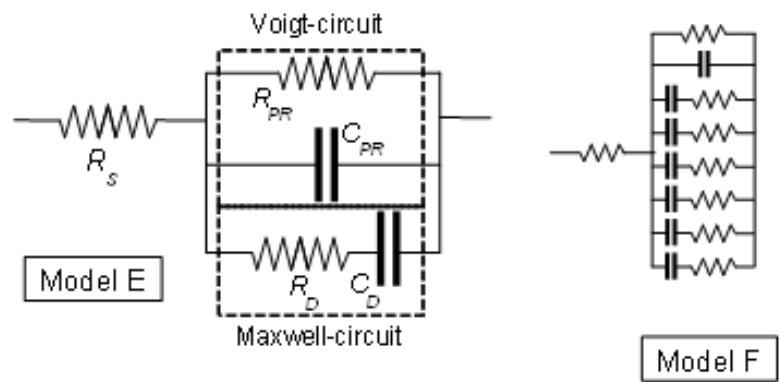

Figure 2: Model E by applying the general scheme and Model F with the series of Maxwell circuits connected in parallel.

tions from it are due to the parameter dispersions and/or mechanistic effects, moreover, all these effects can be fitted using a properly chosen series of EC elements. After due consideration, the model of a Voigt circuit (equivalent to Model A) and a series of Maxwell circuits connected in parallel was chosen (see Fig. 2, Model F). Model F has some important features which have practical advantages; the part of the EC corresponding to Model A, representing the 'ideal' behaviour of the interface, is separated parallel from the rest of the EC. This arrangement results in a linear separation in the compensated admittance:

$$
Y_{\mathrm{C}}=Y_{\mathrm{PR}}+i \omega C_{\mathrm{PR}}+\sum_{k=1}^{K} \frac{i \omega C_{\mathrm{D}, k}}{1+i \omega \tau_{\mathrm{D}, k}}
$$

where $Y_{\mathrm{PR}}=1 / R_{\mathrm{PR}}$ and $C_{\mathrm{PR}}$ denote the principal admittance and capacitance, respectively, $C_{\mathrm{D}, k}$ represents the capacitance and $\tau_{\mathrm{D}, k}$ stands for the time constant of the $k$ th Maxwell circuit connected in parallel. The physical interpretation, performance and limitations of the principal parameters as well as the linearization in general are discussed in detail in previous papers $[36,37]$. In Ref. [36], an equation to determine the principal admittance was published by our group $\left(x=\omega^{2}\right)$ :

$$
\frac{\partial \ln x}{\partial \ln Y_{\mathrm{C}}^{\prime}}=Y_{\mathrm{PR}} \frac{\partial \ln x}{\partial Y_{\mathrm{C}}^{\prime}}+1
$$

The principal admittance which is, under certain conditions, equivalent to the reciprocal of the charge transfer or polarisation resistance, can be determined from a relatively narrow frequency range approximate to or higher than the critical frequency ${ }^{\S}$. This is a serious advantage to any nonlinear model fitting which requires impedance data from a wider and, in particular, a lower frequency range (i.e., lower than the critical frequency) where the adsorption/desorption or transport (diffusion) processes may have a stronger impact on the measured impedance data. In a previous paper [36], Eq. 4 was tested in an

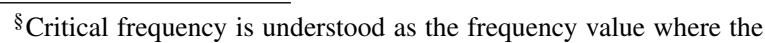
imaginary part of the impedance has a (local) extremum at the 'top' of the 'depressed semicircle.' The accurate value of the critical frequency can be calculated via a quadratic fitting of the nearest points. In some EIS spectra, such an extremum cannot be established.
}

experimental system (quinhydrone redox system in $10 \%$ $\mathrm{HCl}$ ) and, in a more recent paper [37], other methods developed to determine the principal admittance were tested in the quinhydrone as well as three other systems, namely $\mathrm{Fe}, \mathrm{Cu}$ and $\mathrm{COR}$ (see their descriptions in the Experimental section below). In this paper, the testing of Eq. 4 against the latter three systems is presented and discussed.

\section{Experimental}

In order to gain a comprehensive overview concerning the performance of the proposed new parameter evaluation method based on Eq. 4, three test systems were created (the short names, used for identification in the paper, are in brackets):

$\mathrm{Fe}^{3+} / \mathrm{Fe}^{2+}$ redox system $(\mathrm{Fe})$ : metallic iron was dissolved in $10 \% \mathrm{~m} / \mathrm{v} \mathrm{HCl}$ at a concentration of $8 \cdot 10^{-4}$ $\mathrm{mol} / \mathrm{dm}^{3}$ and $\mathrm{FeCl}_{3} \cdot 6 \mathrm{H}_{2} \mathrm{O}$ was added to set the same concentration of $8 \times 10^{-4} \mathrm{~mol} / \mathrm{dm}^{3}$ for $\mathrm{FeCl}_{3}$. Solutions were diluted using $10 \% \mathrm{HCl}$ to the concentrations indicated in Table 1.

The working, reference and counter electrodes were all composed of platinum for this system. The working electrode was a platinum plate with a surface area of 2 $\mathrm{cm}^{2}$, the reference electrode was a larger platinum sheet with a surface area of $5 \mathrm{~cm}^{2}$ and the counter electrode was a platinum net with an approximate surface area of $20 \mathrm{~cm}^{2}$. The potential of the platinum reference electrode was measured before and after the EIS measurements against a saturated $\mathrm{Ag} / \mathrm{AgCl}$ electrode to check if the redox potential of the system was reasonably close to the expected, calculated values.

$\mathrm{Cu}^{2+} / \mathrm{Cu}$ non-corrosive copper/copper ion system $(\mathrm{Cu})$ : the $\mathrm{CuSO}_{4}$ concentrations and the supporting electrolyte are included in Table 1. The working electrode was a cylindrical copper electrode $30 \mathrm{~mm}$ in length and with a diameter of $6 \mathrm{~mm}$ in a teflon holder, while the geometric surface area of the electrode was $5.94 \mathrm{~cm}^{2}$. The reference electrode was composed of copper with the same dimensions and the counter electrode was a copper sheet with a surface area of approximately $20 \mathrm{~cm}^{2}$.

In the corrosive systems (COR), the working electrode was a DIN St 52-type cylindrical steel electrode 30 $\mathrm{mm}$ in length and with a diameter of $6 \mathrm{~mm}$ in a teflon holder, while the geometric surface area of the electrode was $5.94 \mathrm{~cm}^{2}$. The saturated $\mathrm{Ag} / \mathrm{AgCl}$ electrode was used as the reference electrode and a platinum net with an approximate surface area of $20 \mathrm{~cm}^{2}$ as a counter electrode. The electrolyte compositions are shown in Table 1.

All the working electrodes were degreased in acetone. The platinum electrodes were kept in a $10 \% \mathrm{HCl}$ solution for at least 3 hours before the experiment. The copper and steel electrodes were polished with \#400, \#600 and finally \#1000 emery paper, while the copper electrodes were etched in a solution of $20 \% \mathrm{HNO}_{3}$ for 5 minutes. The steel electrodes used for the corrosion experiments 
Table 1: Summary of the parameters and identifiers of the systems and series.

\begin{tabular}{|c|c|c|c|c|c|}
\hline \multicolumn{2}{|c|}{ System } & \multicolumn{2}{|r|}{ Series } & \multirow[t]{2}{*}{ Electrode } & \multirow[t]{2}{*}{ Electrolyte } \\
\hline Abbreviation & Short description & ID & Definition & & \\
\hline $\mathrm{Fe}$ & $\mathrm{Fe}(\mathrm{II}) / \mathrm{Fe}(\mathrm{III})$ redox & $\begin{array}{l}1 \mathrm{E}-4 \\
2 \mathrm{E}-4 \\
4 \mathrm{E}-4 \\
8 \mathrm{E}-4\end{array}$ & $\begin{array}{l}\mathrm{Fe}(\mathrm{II}), \mathrm{Fe}(\mathrm{III}) \\
\text { concentration, } \mathrm{M}\end{array}$ & Platinum & $10 \% \mathrm{HCl}$ \\
\hline $\mathrm{Cu}$ & $\begin{array}{l}\mathrm{Cu} / \mathrm{Cu}(\mathrm{II}) \\
\text { reversible metal }\end{array}$ & $\begin{array}{l}1 \mathrm{E}-4 \\
2 \mathrm{E}-4 \\
4 \mathrm{E}-4 \\
8 \mathrm{E}-4\end{array}$ & $\begin{array}{l}\mathrm{Cu}(\mathrm{II}) \\
\text { concentration, } \mathrm{M}\end{array}$ & $\mathrm{Cu}$ & $1 \mathrm{M} \mathrm{H}_{2} \mathrm{SO}_{4}$ \\
\hline COR & Corrosion system & $\begin{array}{l}\text { COR1 } \\
\text { COR2 } \\
\text { COR3 } \\
\text { COR4 }\end{array}$ & N/A & St-52 steel & $\begin{array}{c}5 \% \mathrm{NaCl}+0.5 \% \mathrm{HAc}^{*} \\
5 \% \mathrm{NaCl}+0.1 \% \mathrm{HCl} \\
1 \% \mathrm{HCl} \\
10 \% \mathrm{HCl}\end{array}$ \\
\hline
\end{tabular}

(system COR) were pre-treated in a $10 \% \mathrm{HCl}$ solution for 5 minutes before being placed in the cell.

All the experiments were carried out in a conventional three-electrode electrochemical cell with a volume of approximately $700 \mathrm{~cm}^{3}$. The experiments were conducted at room temperature and the solutions were not deaerated. All EIS spectra were measured twice and both runs were evaluated. In all three systems ( $\mathrm{Fe}, \mathrm{Cu}$ and $\mathrm{COR}$ ), the electrodes were prepared before the first run and only the electrolytes were replaced for the purpose of measuring the subsequent runs. The parameters and identifiers (used to denote the measurement systems and runs in the discussion of the results) are shown in Table 1. Consecutive runs using the same solution composition are denoted with a -1 or -2 suffix at the end of the IDs, e.g., 1E-4-1 and $1 \mathrm{E}-4-2$ for the $\mathrm{Fe}^{3+}$ and $\mathrm{Fe}^{2+}$ concentrations in the Fe system, respectively, namely $10^{-4} \mathrm{~mol} / \mathrm{dm}^{3}(\mathrm{M})$.

The EIS spectra were measured with a Metrohm Autolab PGSTAT 302N-type potentiostat using NOVA 1.11 software. The amplitude of the applied potential signal was $10 \mathrm{mV}$ (i.e., $20 \mathrm{mV}$ p-p). All spectra were taken within the $10 \mathrm{kHz}-100 \mathrm{mHz}$ range and 20 frequency points were measured per decade over a logarithmically equidistant distribution. In all the experiments, two spectra were measured in order to assess the rate of impedance drifts over time.

The linear fittings were generally very good which involved a high count of $9 \mathrm{~s}$ in the correlation coefficient. In order to avoid the cumbersome counting of the digits, the Precision Factor $P_{R}$ was introduced according to

$$
P_{R}=-\lg \left(1-R^{2}\right)
$$

where $R^{2}$ denotes the square of the correlation coefficient (e.g., if $R^{2}=0.999$, then $P_{R}=3$ ).

\section{Results and Discussion}

The Nyquist diagrams of the $\mathrm{Fe}, \mathrm{Cu}$ and $\mathrm{COR}$ systems are shown in Figs. 3-5, respectively. The corresponding Bode plots are available in Ref. [37]. The three systems exhibit quite different characteristics with regard to how well they fit to the conventional EC parameters and nonlinear least squares methods. The Fe system could be fitted with a Randles circuit including a CPE instead of a double layer capacitance if the diffusion-controlled lowfrequency range was longer and consequently better separated.

This fitting procedure was tried but yielded uncertain results with high parameter errors and strong correlations in the Hessian matrix. In general, the analytic methods do not yield good results if the measurement data do not have ranges where they are mainly sensitive to one parameter only. The measurements below $100 \mathrm{mHz}$ last for quite a lengthy period of time and during this time range, the nonstationarity ('time drift') can produce a significant degree of bias with regard to the measurement data which should be avoided. The $\mathrm{Cu}$ system is a bit worse because the low-frequency range data cannot be interpreted in terms of the conventional EC parameters at all.

The COR system is, however, quite different since it can be fitted with a conventional CPE and charge transfer resistance. The common feature in all three systems is a relatively regular and similar arc in the frequency range higher than the critical frequency (i.e., the frequency where the imaginary part of the impedance has a maximum - this is somewhat unclear in the case of the $\mathrm{Cu}$ system but the regular behaviour exhibited in the highfrequency range is clearly visible). The similarities in and regularities of the higher-frequency range impedance data would suggest that only this range should be used to determine both the double layer capacitance, which is inher- 

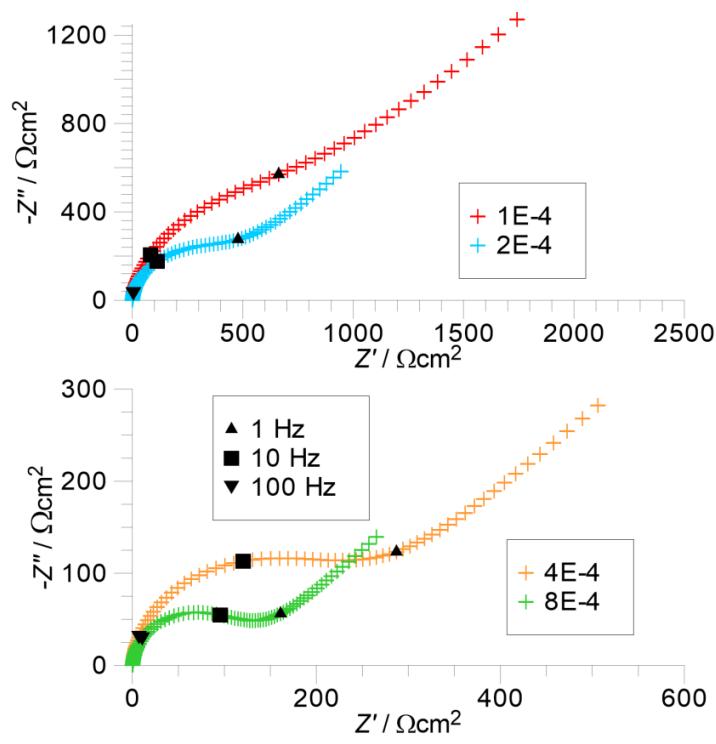

Figure 3: Nyquist plots of the Fe system.

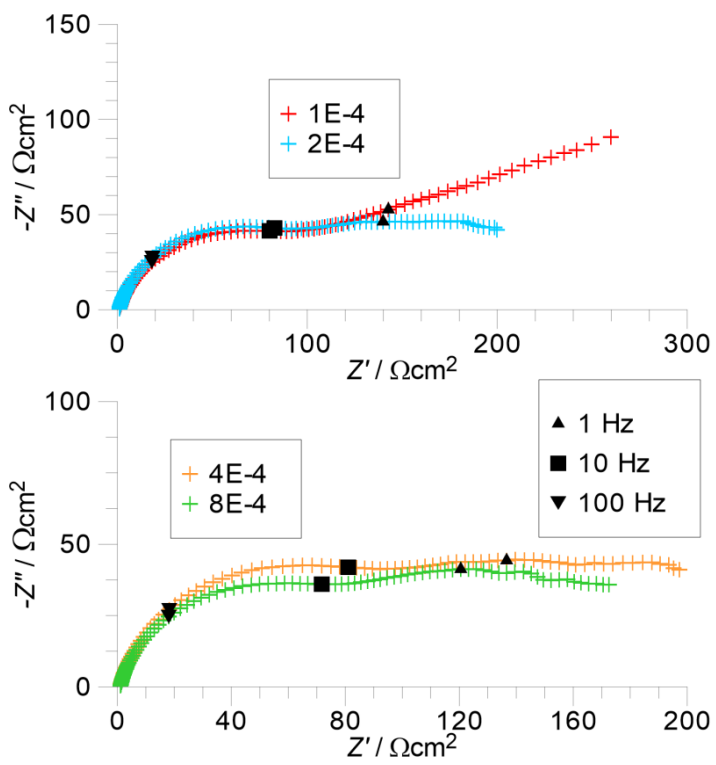

Figure 4: Nyquist plots of the $\mathrm{Cu}$ system.

ently related to the high frequency range, and the charge transfer resistance, which is, in contrast, related to the lower frequency data range. This circumstance requires a more sophisticated approach, which has been outlined in Ref. [36] and is applied in this paper, for the three systems discussed.

Based upon the aforementioned considerations, the importance of developing methods that can determine the charge transfer resistance from the higher frequency data alone is indisputable. The testing of Eq. 4 with the discussed experimental systems is shown in Figs. 6 to 8. The data points are obtained by calculating the gradient $Y_{\mathrm{PR}}$ according to Eq. 4 using the two adjacent points (Method (i)) and by fitting the gradients of the lines to 5 points of both greater and smaller frequencies at each frequency (Method (ii) in Ref. [36]). Therefore, the lines are formed

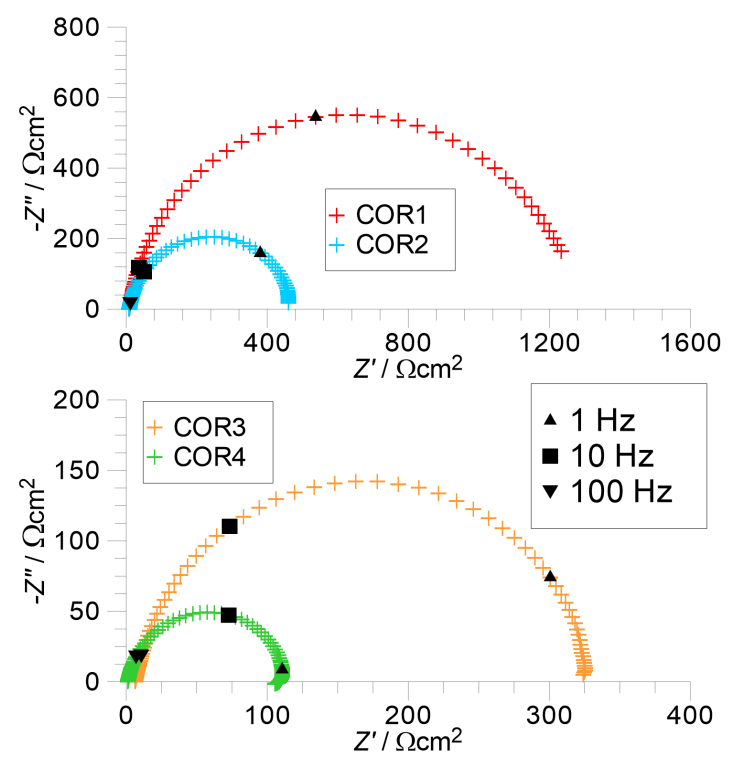

Figure 5: Nyquist plots of the COR system.

by effectively averaging out the points. The curves are strongly dependent on the frequency and, in this case, selecting the most appropriate value to characterize the charge transfer resistance of the system is always an issue. The optimal value of the principal admittance was selected on the basis of the linear-fitting correlation data, moreover, the data set providing the highest correlation coefficient was accepted as the 'real' value. The $P_{R}$ maxima at very high frequencies were not taken into account (see Fig. 6).

By comparing Figs. 3-5 with Figs. 6-8, it can be concluded that the more regular (depressed) semicircles are formed in the Nyquist diagram, the more established principal admittance data can be obtained from Eq. 4 . However, if regular semicircles are formed by a system, then the practical usefulness of the proposed evaluation is strongly limited because in such cases, conventional evaluation methods can generally be applied. This is the case with the COR system. The application of the method is necessitated in systems like that of $\mathrm{Fe}$ and $\mathrm{Cu}$ where conventional nonlinear fitting methods or any other simple methods (e.g. the graphical determination of $\left.R_{\mathrm{CT}}[36,37]\right)$ cannot be used at all or with only a very limited degree of precision. In order to also test the versatility of the method in such systems, the principal admittance values determined via Method (ii) were compared to those calculated by Eq. 13 in Ref. [37] $]^{\text {Il }}$ for the three systems investigated in this paper and also for the quinhydrone $(\mathrm{QH})$ system published in the previous paper [36] on the subject, the results of which are presented in Fig. 9. According to the results, a fairly good correlation coefficient is obtained which indicates that both methods, namely Method (ii) and Eq. 13, calculate the same phys-

\footnotetext{
IT The derivation leading to Eq. 13 (in Ref. [37]) is also based on Eq. 3 (in this paper) but includes specific mathematical transformations based on the elimination of the dispersion parameters from the respective equations.
} 


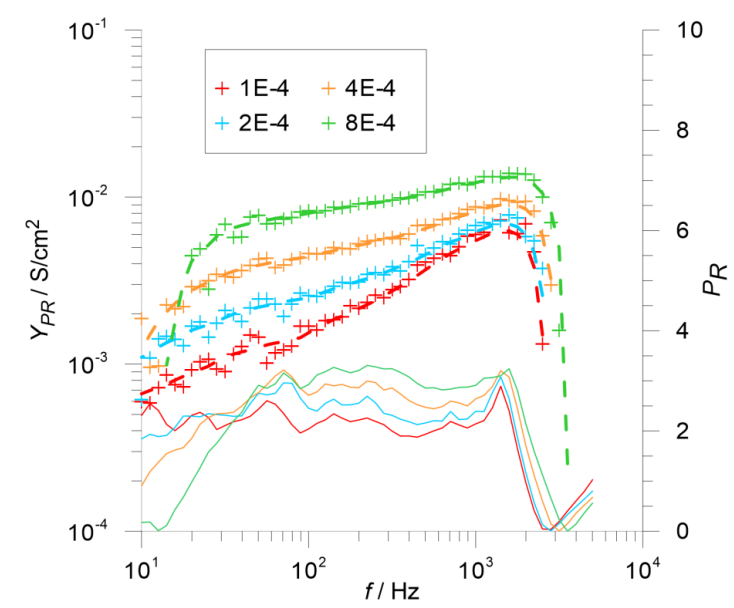

Figure 6: Principal admittance of the Fe system calculated by Eq. 4 using Method (i) (points) and Method (ii) (dotted lines). Solid lines indicate the $P_{R}$ values obtained using Method (ii).

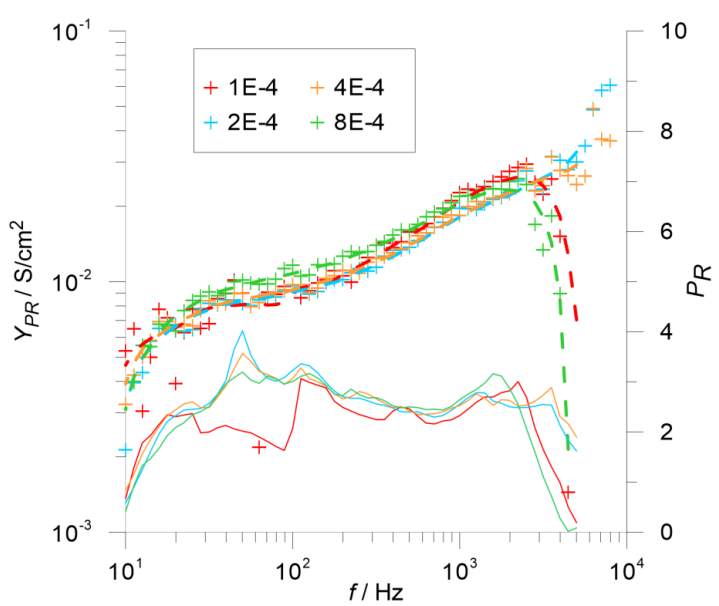

Figure 7: Principal admittance of the $\mathrm{Cu}$ system calculated by Eq. 4 using Method (i) (points) and Method (ii) (dotted lines). Solid lines indicate the $P_{R}$ values obtained using Method(ii).

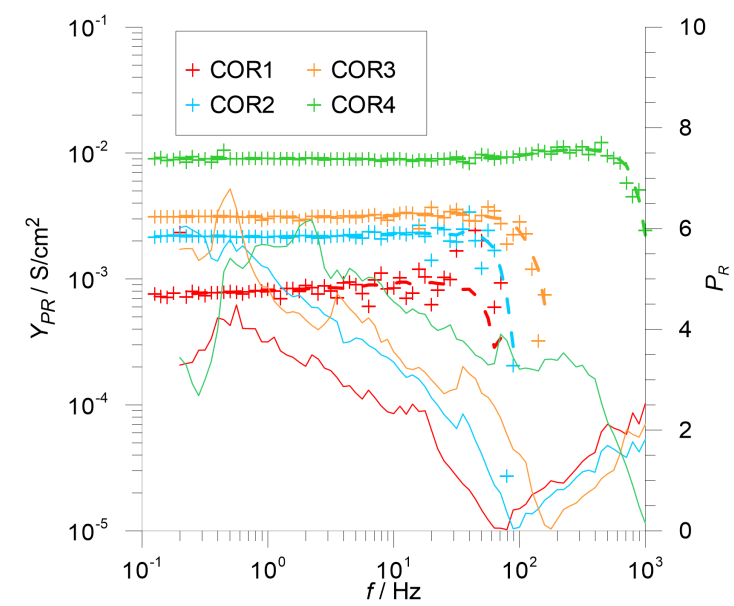

Figure 8: Principal admittance of the COR system calculated by Eq. 4 using Method (i) (points) and Method (ii) (dotted lines). Solid lines indicate the $P_{R}$ values obtained using Method(ii).

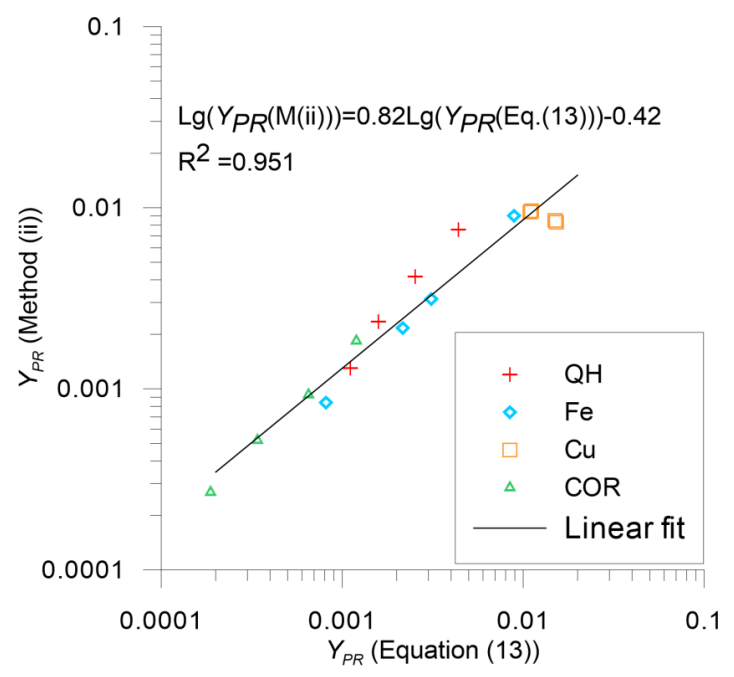

Figure 9: Correlation between the logarithms of the principal admittance obtained by Method(ii) using Eqs. 4 and 13 in Ref. [37]. The linear fit was calculated from the logarithms of the principal admittance data. The QH (quinhydrone) data were taken from Ref. [36].

ical quantity.

The data in Fig. 9 prove that both methods are applicable for the determination of the principal admittance. The question that is typically raised in similar situations is which method is better. In our opinion, it is still too early to make such a judgement given that in Ref. [37] several additional equations were also published to calculate the principal admittance. Some of these equations differ in terms of their initial physical considerations (compare Eq. 4 in this paper and Eq. 13) in Ref. [37]], while others differ only as far as the weighting of data points is concerned (compare Eq. 8 with 8a or Eq. 13 with Eq. 14 in Ref. [37]).

It is recommended that these methods should be applied especially in those cases when the impedance data in the low-frequency range (typically below the critical frequency) are not interpretable or cannot be fitted for some reason (e.g., the applicable EC cannot be assessed unambiguously). In such cases, by applying two or more of the proposed equations, the uncertainty in this determination can be decreased by only utilizing parameters, which are sufficiently close to one another.

\section{Conclusions}

A recent method developed to determine the principal admittance was tested on electrochemical redox and corrosion systems in order to check and compare the performances of the methods. The characteristics of the impedance with regard to such electrochemical systems exhibited features which often make conventional nonlinear fitting methods unusable or at least inaccurate. The new method was applied to determine the principal admittance from the impedance data obtained in the higher frequency range which exhibit a more regular shape but 
are not applicable to conventional fitting methods alone. By comparing this new method with another also newly developed method (Eq. 13 in Ref. [37]), a strong correlation between the parameters measured using the two methods was observed, which proves that both of them are suitable for determining the principal admittance of systems which exhibit similarly regular behaviour within the higher frequency range.

\section{Acknowledgement}

Present article was published in the frame of the project GINOP-2.3.2-15-2016-0053 ('Development of engine fuels with high hydrogen content in their molecular structures (contribution to sustainable mobility)').

\section{REFERENCES}

[1] Cole K.S.; Cole, R.H.: Dispersion and absorption in dielectrics 1 . Alternating current characteristics, J. Chem. Phys., 1941, 9(4), 341-351 DOI: 10.1063/1.1750906

[2] Cole K.S.; Cole, R.H.: Dispersion and absorption in dielectrics 2. Direct current characteristics, J. Chem. Phys., 1942, 10(2), 98-105 DOI: 10.1063/1.1723677

[3] Brug, G.J.; van den Eeden, A.L.G.; SluytersRehbach, M.; Sluyters, J.H.: The analysis of electrode impedances complicated by the presence of a constant phase element, J. Electroanal. Chem., 1984, 176(1-2), 275-295 DOI: 10.1016/S00220728(84)80324-1

[4] Macdonald, D.D.: Review of mechanistic analysis by electrochemical impedance spectroscopy, Electrochim. Acta, 1990, 35(10), 1509-1525 DOI: 10.1016/0013-4686(90)80005-9

[5] Lasia, A.; Rami, A.: Kinetics of hydrogen evolution on nickel electrodes, J. Electroanal. Chem. Interfacial Electrochem., 1990, 294(1-2), 123-141 DOI: 10.1016/0022-0728(90)87140-F

[6] Antańo-Lopez, R.; Keddam, M.; Takenouti, H.: A new experimental approach to the time-constants of electrochemical impedance: frequency response of the double layer capacitance, Electrochim. Acta, 2001, 46(24-25), 3611-3617 DOI: 10.1016/S00134686(01)00640-5

[7] Hu, J.-M.; Zhang, J.-Q.; Cao, Ch.-N.: Oxygen evolution reaction on $\mathrm{IrO}_{2}$-based $\mathrm{DSA}^{\circledR}$ type electrodes: kinetics analysis of Tafel lines and EIS, Int. J. Hydrog. Energy, 2004, 29, 791-797 DOI: 10.1016/j.ijhydene.2003.09.007

[8] Lockett, V.; Sedev, R.; Ralston, J.; Horne, M.; Rodopoulos, T.: Differential capacitance of the electrical double layer in imidazolium-based ionic liquids: influence of potential, cation size, and temperature, J. Phys. Chem. C, 2008, 112, 7486-7495 DOI: 10.1021/jp7100732
[9] Nyikos, L.; Pajkossy,T.: Electrochemistry at fractal interfaces: the coupling of $a c$ and $d c$ behavior at irregular electrodes, Electrochim. Acta, 1990, 35(10), 1567-1572 DOI: 10.1016/0013-4686(90)80011-C

[10] Pajkossy, T.: Impedance of rough capacitive electrodes, J. Electroanal. Chem., 1994, 364(1-2), 111125 DOI: 10.1016/0022-0728(93)02949-I

[11] Lasia, A.: Impedance of porous electrodes, $J$. Electroanal. Chem., 1995, 397(1-2), 27-33 DOI: 10.1016/0022-0728(95)04177-5

[12] Kerner, Zs.; Pajkossy, T.: On the origin of capacitance dispersion of rough electrodes, Electrochim. Acta, 2000, 46(2-3), 207-211 DOI: 10.1016/S00134686(00)00574-0

[13] Jović, V.D.; Jović, B.M.: EIS and differential capacitance measurements onto single crystal faces in different solutions Part $\mathrm{I}: \mathrm{Ag}(111)$ in $0.01 \mathrm{M}$ $\mathrm{NaCl}$, J. Electroanal. Chem., 2003, 541, 1-11 DOI: 10.1016/S0022-0728(02)01309-8

[14] Hirschorn, B.; Orazem, M.E.; Tribollet, B.; Vivier, V.; Frateur, I.; Musiani, M.: Determination of effective capacitance and film thickness from constantphase-element parameters, Electrochim. Acta, 2010, 55(21), 6218-6227 DOI: 10.1016/j.electacta.2009.10.065

[15] Jüttner, K.; Lorenz, W.J.; Kendig, M.W.; Mansfeld, F.: Electrochemical impedance spectroscopy on 3D inhomogeneous surfaces, J. Electrochem. Soc., 1988, 135, 332-339 DOI: 10.1149/1.2095610

[16] Hack, H.P.; Scully, J.R.: Defect area determination of organic coated steels in seawater using the breakpoint frequency method, J. Electrochem. Soc., 1991, 138(1), 33-40 DOI: 10.1149/1.2085574

[17] Rammelt, U.; Reinhard, G.: Application of electrochemical impedance spectroscopy (EIS) for characterizing the corrosion-protective performance of organic coatings on metals, Prog. Org. Coat., 1992, 21(2-3), 205-226 DOI: 10.1016/0033-0655(92)87005-U

[18] Tan, Y.J.; Bailey, S.; Kinsella, B.: An investigation of the formation and destruction of corrosion inhibitor films using electrochemical impedance spectroscopy (EIS), Corros. Sci., 1996, 38(9), 15451561 DOI: 10.1016/0010-938X(96)00047-9

[19] Macdonald, D.D.; Sikora, E.; Engelhardt, G.: Characterizing electrochemical systems in the frequency domain, Electrochim. Acta, 1998, 43(1-2), 87-107 DOI: 10.1016/S0013-4686(97)00238-7

[20] Bommersbach, P.; Alemany-Dumont, C.; Millet, J.P.; Normand, B.: Formation and behaviour study of an environment-friendly corrosion inhibitor by electrochemical methods, Electrochim. Acta, 2005, 51(6), 1076-1084 DOI: 10.1016/j.electacta.2005.06.001

[21] Baril, G.; Galicia, G.; Deslouis, C.; Pébčre, N.; Tribollet, B.; Vivier, V.: An impedance investigation of the mechanism of pure magnesium corrosion in sodium sulfate solutions, J. Electrochem. Soc., 2007, 154(2), C108 DOI: 10.1149/1.2401056

[22] Levi, M.D.; Salitra, G.; Markovsky, B.; Teller, H.; Aurbach, D.; Heider, U.; Heider, L.: Solid- 
state electrochemical kinetics of Li-ion intercalation into $\mathrm{Li}_{1-x} \mathrm{CoO}_{2}$ : Simultaneous application of electroanalytical techniques SSCV, PITT, and EIS, J. Electrochem. Soc., 1999, 146(4), 1279-1289 DOI: 10.1149/1.1391759

[23] Wang, C.; Appleby, A.J.; Little, F.E.: Electrochemical study on nano-Sn, $\mathrm{Li}_{4} .4 \mathrm{Sn}$ and $\mathrm{AlSi}_{0} .1$ powders used as secondary lithium battery anodes, J. Power Sources, 2001, 93(1-2), 174-185 DOI: 10.1016/S03787753(00)00576-0

[24] Li, Z.E.; Du, F.; Bie, X.; Zhang, D.; Cai, Y.; Cui, X.; Wang, C.; Chen, G.; Wei, Y.: Electrochemical kinetics of the $\mathrm{Li}\left[\mathrm{Li}_{0.23} \mathrm{Co}_{0.3} \mathrm{Mn}_{0.47}\right] \mathrm{O}_{2}$ cathode material studied by GITT and EIS, J. Phys. Chem. C, 2010, 114, 22751-22757 DOI: 10.1021/jp1088788

[25] Tang, K.; Yu, X.; Sun, J.; Li, H.; Huang, X.: Kinetic analysis on $\mathrm{LiFePO}_{4}$ thin films by CV, GITT, and EIS, Electrochim. Acta, 2011, 56(13), 4869-4875 DOI: 10.1016/j.electacta.2011.02.119

[26] Niya, S.M.R.; Hoorfar, M.: Study of proton exchange membrane fuel cells using electrochemical impedance spectroscopy technique - A review, J. Power Sources, 2013, 240, 281-293 DOI: 10.1016/j.jpowsour.2013.04.011

[27] Morin, A.; Peng, Z.; Jestin, J.; Detrez, M.; Gebel, G.: Water management in proton exchange membrane fuel cell at sub-zero temperatures: An in operando SANS-EIS coupled study, Solid State Ion., 2013, 252, 56-61 DOI: 10.1016/j.ssi.2013.07.010

[28] Zhiani, M.; Majidia, S.; Silva, W.B.; Gharibi, H.: Comparison of the performance and EIS (electrochemical impedance spectroscopy) response of an activated PEMFC (proton exchange membrane fuel cell) under low and high thermal and pressure stresses, Energy, 2016, 97, 560-567 DOI: 10.1016/j.energy.2015.12.058

[29] Oliveira, J.L.; Skilbred, A.W.B.; Loken, A.; Henriques, R.R.; Soares, B.G.: Effect of accelerated ageing procedures and flash rust inhibitors on the anti-corrosive performance of epoxy coatings: EIS and dynamic-mechanical analysis, Prog. Org. Coat., 2021, 159, 106387 DOI: 10.1016/j.porgcoat.2021.106387

[30] Martinez, S.; Šoić, I.; Špada, V.: Unified equivalent circuit of dielectric permittivity and porous coating formalisms for EIS probing of thick industrial grade coatings, Prog. Org. Coat., 2021, 153, 106155 DOI: 10.1016/j.porgcoat.2021.106155

[31] Hinderliter, B.R.; Croll, S.G.; Tallman, D.E.; Su, Q.; Bierwagen, G.P.: Interpretation of EIS data from accelerated exposure of coated metals based on modeling of coating physical properties, Electrochim. Acta, 2006, 51(21), 4505-4515 DOI: 10.1016/j.electacta.2005.12.047

[32] Lukács, Z.: Evaluation of model and dispersion parameters and their effects on the formation of constant-phase elements in equivalent circuits, J. Electroanal. Chem., 1999, 464(1), 68-75 DOI: 10.1016/S0022-0728(98)00471-9

[33] Agarwal, P.; Orazem, M.E.; Garcia-Rubio, L.H.: Measurement models for electrochemical impedance spectroscopy: I . Demonstration of applicability, J. Electrochem. Soc., 1992, 139(7), 1917-1927 DOI: 10.1149/1.2069522

[34] Fletcher, S.: Tables of degenerate electrical networks for use in the equivalent-circuit snalysis of electrochemical systems, J. Electrochem. Soc., 1994, 141(7), 1823-1826 DOI: 10.1149/1.2055011

[35] Harrington, D.A.; van den Driessche, P.: Mechanism and equivalent circuits in electrochemical impedance spectroscopy, Electrochim. Acta, 2011, 56(23), 8005-8013 DOI: 10.1016/j.electacta.2011.01.067

[36] Lukács, Z.; Kristóf, T.: A generalized model of the equivalent circuits in the electrochemical impedance spectroscopy, Electrochim. Acta, 2020, 363, 137199 DOI: 10.1016/j.electacta.2020.137199

[37] Lukács, Z.; Baccilieri, D.; Hancsók, J.; Kristóf, T.: A dispersion-invariant model of the electrochemical impedance, Electrochim. Acta, 2021, 390, 138828 DOI: $10.1016 /$ j.electacta.2021.138828 